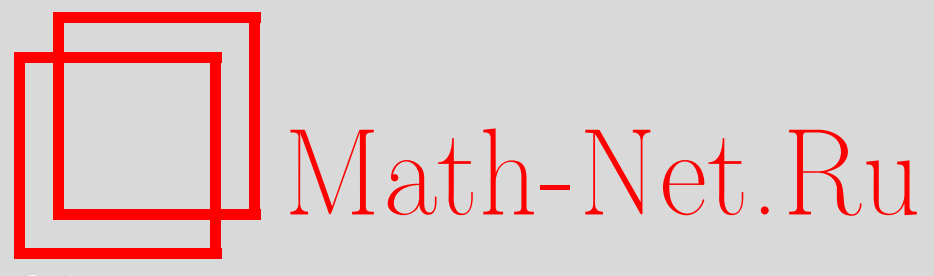

Т. Оздоган, М. Орбей, Формула разложения для произведения двух нормированных присоединенных функций Лежандра и ее преимущества при вычислении многоцентровых интегралов, Матем. заметки, 2006, том 80, выпуск 1, 144-148

DOI: https://doi.org/10.4213/mzm2791

Использование Общероссийского математического портала Math-Net.Ru подразумевает, что вы прочитали и согласны с пользовательским соглашением http://www.mathnet.ru/rus/agreement

Параметры загрузки:

IP : 52.23 .180 .231

26 апреля 2023 г., 17:06:00

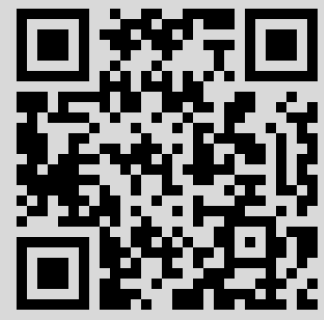




\section{ФОРМУЛА РАЗЛОЖЕНИЯ ДЛЯ ПРОИЗВЕДЕНИЯ ДВУХ НОРМИРОВАННЫХ ПРИСОЕДИНЕННЫХ ФУНКЦИЙ ЛЕЖАНДРА И ЕЕ ПРЕИМУЩЕСТВА ПРИ ВЫЧИСЛЕНИИ МНОГОЦЕНТРОВЫХ ИНТЕГРАЛОВ}

\section{Т. Оздоган, М. Орбей}

1. Введение. В настоящее время нет необходимости объяснять, какую роль играет уравнение Шрёдингера в науке и технологии. Фактически, любой природный феномен может быть понят, если удается решить соответствующие уравнения Шрёдингера. K сожалению, уравнение Шрёдингера может быть решено только аналитически и только в некоторых частных случаях. Следовательно, при изучении более сложных систем, а именно, многоэлектронных систем, у нас нет другого пути, как только обратиться к методам аппроксимации; в области квантовой химии наиболее часто используются вариационные методы. Естественно ожидать, что пробные функции, которые применяются в этом процессе, должны быстро сходиться к точному решению, а также удовлетворять условию заостренной точки возврата Като на ядрах [1] и условию экспоненциального убывания на бесконечности [2]. Таким образом, орбитали Слетеровского типа имеют фундаментальное значение при изучении различных свойств молекул, так как они удовлетворяют выше упомянутым критериям. С другой стороны, в процессе вычисления электронной структуры многоэлектронных систем, нужно точно и эффективно оценивать многоцентровые интегралы по орбиталям Слетеровского типа. Среди известных в литературе методов вычисления многоцентровых интегралов по орбиталям Слетеровского типа, наиболее известен метод эллиптических координат, который применяется уже более столетия и который позволяет получить надежные результаты для атомов и молекул [3]. Для достижения эффективности, точности и скорости сходимости необходимо иметь полезную формулу для вывода формулы разложения для произведения двух нормированных присоединенных функций Лежандра в эллиптической системе координат.

Данная статья имеет двоякую цель: во-первых, получить аналитическую формулу разложения для произведения двух нормированных присоединенных функций Лежандра в эллипсоидальных координатах, а во-вторых, обсудить основные преимущества предлагаемой формулы при оценке многоцентровых интегралов по орбиталям Слетеровского типа.

2. Формула разложения для произведения двух нормированных присоединенных функций Лежандра с разными центрами. Для вычисления произвольных многоцентровых многоэлектронных интегралов по орбиталям Слетеровского типа [4], [5] требуется формула для разложения произведения двух нормированных присоединенных функций Лежандра с центрами в точках $a$ и $b$,

$$
T^{l \lambda, l^{\prime} \lambda}\left(\theta_{a}, \theta_{b}\right)=P_{l \lambda}\left(\cos \theta_{a}\right) P_{l^{\prime} \lambda}\left(\cos \theta_{b}\right) .
$$

В работе Ясуи и Саика [6] нормированные присоединенные функции Лежандра выражены аналитически через факториалы. Используя известные свойства биномиальных коэффициентов, мы получили следующее выражение для нормированных присоединенных функций Лежандра [7]:

$$
P_{l m}(\cos \theta)=(1)^{(|m| m) / 2} \sum_{k} C_{l m}^{k}(\sin \theta)^{2 k+m}(\cos \theta)^{l(2 k+m)},
$$


где $\frac{1}{2}(|m|-m) \leqslant k \leqslant E\left(\frac{l-m}{2}\right)$,

$$
\begin{gathered}
E\left(\frac{n}{2}\right)=\frac{n}{2}-\frac{1}{4}\left(1-(-1)^{n}\right), \\
C_{l m}^{k}=\frac{(-1)^{k}}{2^{2 k+m}}\left[\frac{2 l+1}{2} F_{l-k}(l+m) F_{k+m}(l-k) F_{2 k}(l-m) F_{k}(2 k)\right]^{1 / 2} .
\end{gathered}
$$

Чтобы получить выражение для произведения двух нормированных присоединенных функций Лежандра, нам понадобятся следующие формулы, связывающие сферические полярные координаты и эллипсоидные координаты с центрами на атомах $A$ и $B$, лежащих на расстоянии $R$ друг от друга:

$$
\begin{aligned}
r_{a} & =\frac{R}{2}(\mu+\nu), & \cos \theta_{a} & =\frac{1+\mu \nu}{\mu+\nu}, \\
r_{b} & =\frac{R}{2}(\mu-\nu), & \sin \theta_{a} & =\frac{\left[\left(\mu^{2}-1\right)\left(1-\nu^{2}\right)\right]^{1 / 2}}{\mu+\nu}, \\
\cos \theta_{b} & =\frac{1-\mu \nu}{\mu-\nu}, & \sin \theta_{b} & =\frac{\left[\left(\mu^{2}-1\right)\left(1-\nu^{2}\right)\right]^{1 / 2}}{\mu-\nu} .
\end{aligned}
$$

Подставляя соотношения (2)-(5) в формулу (1), получаем следующее выражение для произведения двух нормированных присоединенных функций Лежандра в эллипсоидальных координатах:

$$
\begin{aligned}
& T^{l \lambda, l^{\prime} \lambda}(\mu, \nu)=P_{l \lambda}\left(\frac{1+\mu \nu}{\mu+\nu}\right) P_{l^{\prime} \lambda}\left(\frac{1-\mu \nu}{\mu-\nu}\right) \\
& =\sum_{k} C_{l \lambda}^{k}\left(\frac{\left[\left(\mu^{2}-1\right)\left(1-\nu^{2}\right)\right]^{1 / 2}}{\mu+\nu}\right)^{2 k+\lambda}\left(\frac{1+\mu \nu}{\mu+\nu}\right)^{l-(2 k+\lambda)} \\
& \quad \times \sum_{k^{\prime}} C_{l^{\prime} \lambda}^{k^{\prime}}\left(\frac{\left[\left(\mu^{2}-1\right)\left(1-\nu^{2}\right)\right]^{1 / 2}}{\mu-\nu}\right)^{2 k^{\prime}+\lambda}\left(\frac{1-\mu \nu}{\mu-\nu}\right)^{l^{\prime}-\left(2 k^{\prime}+\lambda\right)} \\
& =\sum_{k k^{\prime}} C_{l \lambda}^{k} C_{l^{\prime} \lambda}^{k^{\prime}} \frac{\left[\left(\mu^{2}-1\right)\left(1-\nu^{2}\right)\right]^{k+k^{\prime}+\lambda}}{(\mu+\nu)^{l}(\mu-\nu)^{l^{\prime}}}(1+\mu \nu)^{l-(2 k+\lambda)}(1-\mu \nu)^{l^{\prime}-\left(2 k^{\prime}+\lambda\right)} .
\end{aligned}
$$

Применяя тождество

$$
\left(\mu^{2}-1\right)\left(1-\nu^{2}\right)=(\mu+\nu)^{2}-(1+\mu \nu)^{2}
$$

и хорошо известное биномиальное разложение

$$
(\mu+\nu)^{N}=\sum_{m=0}^{N} F_{m}(N) \mu^{N-m} \nu^{m},
$$

получаем

$$
\begin{aligned}
{\left[\left(\mu^{2}-1\right)\left(1-\nu^{2}\right)\right]^{k+k^{\prime}+\lambda} } & =\left[(\mu+\nu)^{2}-(1+\mu \nu)^{2}\right]^{k+k^{\prime}+\lambda} \\
& =\sum_{u=0}^{k+k^{\prime}+\lambda}(-1)^{u} F_{u}\left(k+k^{\prime}+\lambda\right)(\mu+\nu)^{2\left(k+k^{\prime}+\lambda\right)-2 u}(1+\mu \nu)^{2 u} .
\end{aligned}
$$

Подставляя (9) в (6), находим

$$
\begin{aligned}
& T^{l \lambda, l^{\prime} \lambda}(\mu, \nu) \\
& \quad=\sum_{k k^{\prime}} C_{l \lambda}^{k} C_{l^{\prime} \lambda}^{k^{\prime}} \sum_{u=0}^{k+k^{\prime}+\lambda}(-1)^{u} F_{u}\left(k+k^{\prime}+\lambda\right) \frac{(1+\mu \nu)^{l-(2 k+\lambda)+2 u}(1-\mu \nu)^{l^{\prime}-\left(2 k^{\prime}+\lambda\right)}}{(\mu+\nu)^{l-2\left(k+k^{\prime}+\lambda\right)+2 u}(\mu-\nu)^{l^{\prime}}} .
\end{aligned}
$$


Наконец, используя хорошо известное соотношение [8]

$$
(\mu+\nu)^{N}(\mu-\nu)^{N^{\prime}}=\sum_{m=0}^{N+N^{\prime}} F_{m}\left(N, N^{\prime}\right) \mu^{N+N^{\prime}-m} \nu^{m}
$$

в формуле (10), получаем

$$
\begin{aligned}
& (1+\mu \nu)^{l-(2 k+\lambda)+2 u}(1-\mu \nu)^{l^{\prime}-\left(2 k^{\prime}+\lambda\right)} \\
& \quad=\sum_{s=0}^{l+l^{\prime}-2\left(k+k^{\prime}+\lambda\right)+2 u} F_{s}\left(l-2 k-\lambda+2 u, l^{\prime}-2 k^{\prime}-\lambda\right)(\mu \nu)^{s} .
\end{aligned}
$$

Подставляя выражение (12) в (10), получаем формулу разложения для произведения двух нормированных присоединенных функций Лежандра в виде

$$
T^{l \lambda, l^{\prime} \lambda}(\mu, \nu)=\sum_{k, k^{\prime}} \sum_{u, s} a_{u s}^{k k^{\prime}}\left(l \lambda, l^{\prime} \lambda\right) \frac{(\mu \nu)^{s}}{(\mu+\nu)^{l-2\left(k+k^{\prime}+\lambda\right)+2 u}(\mu-\nu)^{l^{\prime}}},
$$

где коэффициенты разложения имеют вид

$$
a_{u s}^{k k^{\prime}}\left(l \lambda, l^{\prime} \lambda\right)=C_{l \lambda}^{k} C_{l^{\prime} \lambda}^{k^{\prime}}(-1)^{u} F_{u}\left(k+k^{\prime}+\lambda\right) F_{s}\left(l-2 k-\lambda+2 u, l^{\prime}-2 k^{\prime}-\lambda\right),
$$

а индексы суммирования $k, k^{\prime}, u$ и $s$ пробегают следующие интервалы:

$$
\begin{array}{ll}
0 \leqslant k \leqslant E\left(\frac{l-\lambda}{2}\right), & 0 \leqslant k^{\prime} \leqslant E\left(\frac{l^{\prime}-\lambda}{2}\right), \\
0 \leqslant u \leqslant\left(k+k^{\prime}+\lambda\right), & 0 \leqslant s \leqslant\left(l+l^{\prime}\right)-2\left(k+k^{\prime}+\lambda\right)+2 u .
\end{array}
$$

Функция $F_{m}\left(N, N^{\prime}\right)$, стоящая в выражениях $(11),(12)$ и (14), определена в работе [8].

Подобные выражения для произведения двух нормированных присоединенных функций Лежандра в эллипсоидальных координатах можно найти и в других работах (см. (A.12) в [8] и (10) в [9]). Формулы в этих работах содержат факториалы, тогда как наша формула содержит биномы, что дает очень полезные рекуррентные соотношения и, следовательно, позволяет вычислять многоцентровые интегралы с достаточной скоростью и точностью. В тоже время, в работе [10] рассматривается взаимосвязь между процедурами, представленными в этих работах, и, следовательно, работа [10] имеет большое значение для обоснования нашего метода.

3. Основные преимущества нашего метода вычисления многоцентровых интегралов. Из литературы хорошо известно, что эффективность (скорость сходимости и точность) компьютерных вычислений для многоцентровых интегралов по орбиталям Слетеровского типа уменьшается с ростом значений квантовых чисел [11], а также для значений, близких к значениям орбитального и магнитного квантовых чисел $(l, m)[12]$. Одним из примеров вышеупомянутых задач этого типа является цифровая эрозия для двухцентровых интегралов перекрывания, которая обсуждается в прекрасной работе Барнета [11].

Проверяя наш метод на молекулярных интегралах, мы сконцентрировали наше внимание на вычислении двухцентровых интегралов по орбиталям Слетеровского типа. Вопервых, мы сравнили наши результаты, полученные для двухцентровых интегралов перекрывания, с результатами, полученными в работе Барнета [11] при больших значениях квантовых чисел, и увидели, что наименьшая точность составляет 14 десятичных знаков после запятой. С другой стороны, обнаружены некоторые расхождения с результатами работы [12], в которой подобная процедура используется при больших значениях 
ТАБлицА 1. Сравнение значений двухцентровых интегралов перекрытия (в атомной системе единиц) и необходимого времени работы ЦП для двух различных процедур

\begin{tabular}{|c|c|c|c|c|c|c|c|}
\hline$n$ & $l$ & $n^{\prime}$ & $\lambda$ & $p$ & $l$ & Работа $[13]$ & Щ* (мсек) $^{*}$ \\
\hline 12 & 7 & 12 & 7 & 3 & 15 & 0.3 & $2.29354178100625 \mathrm{E}-02$ \\
\hline 17 & 8 & 14 & 6 & 4 & 30 & 0.8 & $9.13905848808666 \mathrm{E}-07$ \\
\hline 21 & 16 & 19 & 13 & 11 & 0.5 & 0.2 & $9.49677653441923 \mathrm{E}-05$ \\
\hline 26 & 10 & 26 & 10 & 8 & 40 & 0.6 & $-6.16109990069403 \mathrm{E}-05$ \\
\hline
\end{tabular}

\begin{tabular}{|c|c|c|c|c|c|c|c|c|}
\hline$n$ & $l$ & $n^{\prime}$ & $\lambda$ & $p$ & $l$ & Работа $[4]$ & Ц* $^{*}$ мсек) & \\
\hline 12 & 7 & 12 & 7 & 3 & 15 & 3560 & $2.29354178095047 \mathrm{E}-02$ & 10 \\
\hline 17 & 8 & 14 & 6 & 4 & 30 & 1390 & $9.14266771067075 \mathrm{E}-07$ & 14 \\
\hline 21 & 16 & 19 & 13 & 11 & 0.5 & 10180 & $9 . \underline{49677653399829 \mathrm{E}-05}$ & 95 \\
\hline 26 & 10 & 26 & 10 & 8 & 40 & 8080 & $-6 . \underline{18053677005182 \mathrm{E}-05}$ & 48 \\
\hline
\end{tabular}

Время работы ЦП дано в миллисекундах (мсек); фазовый коэффициент не учитывается в [13].

ТАБлицА 2. Сравнение числа членов, возникающих в формуле для $T^{l \lambda, l^{\prime} \lambda}(\mu, \nu)$, для двух различных процедур

\begin{tabular}{|cc|cc|c|c|}
\hline$l$ & $\lambda$ & $l^{\prime}$ & $\lambda$ & \multicolumn{2}{|c|}{ Число членов в $T^{l \lambda, l^{\prime} \lambda}(\mu, \nu)$} \\
\cline { 5 - 6 } & & & & ф-ла (9) в $[4]$ & ф-ла $(10)$ в $[13]$ \\
\hline 1 & 1 & 1 & 1 & 4 & 6 \\
\hline 2 & 1 & 2 & 1 & 8 & 24 \\
\hline 2 & 2 & 2 & 2 & 9 & 15 \\
\hline 3 & 3 & 3 & 3 & 16 & 28 \\
\hline 4 & 4 & 4 & 4 & 25 & 45 \\
\hline 5 & 5 & 5 & 5 & 36 & 66 \\
\hline 6 & 6 & 6 & 6 & 49 & 91 \\
\hline
\end{tabular}

квантовых чисел. Расхождения особенно велики в случае значений, почти равных орбитальному и магнитному квантовым числам (таблица 1), тогда как хорошее совпадение получается для двухцентровых интегралов перекрытия при небольших значениях квантовых чисел. Итак, из таблицы 1 видно, что время компьютерных вычислений (время работы ЦП) в нашем случае значительно меньше, чем время компьютерных вычислений в работе [13]. По-видимому, одной из причин этого являются слагаемые, появляющиеся в формуле разложения для произведения двух нормированных присоединенных функций Лежандра. Число слагаемых, возникающих в формуле разложения произведения двух нормированных присоединенных функций Лежандра в нашей процедуре [4] и в формуле (10) работы [9], дано в таблице 2 для некоторых квантовых наборов $\left(l \lambda, l^{\prime} \lambda\right)$.

Из таблицы 2 видно, что в нашей процедуре в формуле для $T^{l \lambda, l^{\prime} \lambda}(\mu, \nu)$ число возникающих слагаемых меньше, чем в формуле (10) в процедуре работы [9], что понижает время компьютерных вычислений двухцентровых интегралов перекрывания.

Следовательно, наша процедура вычисления произведения двух нормированных присоединенных функций Лежандра может применяться для вычисления произвольных многоцентровых многоэлектронных молекулярных интегралов по орбиталям Слетеровского типа без потери точности и с высокой скоростью. В этом отношении, наша работа является продолжением исследований по применению произведения двух нормированных присоединенных функций Лежандра в общей теории (см. нашу предыдущую работу [5]) для вычисления многоцентровых многоэлектронных интегралов по орбита- 
лям Слетеровского типа. Некоторые предварительные результаты будут опубликованы в ближайшее время.

\section{СПИСОК ЦИТИРОВАННОЙ ЛИТЕРАТУРЫ}

[1] T. Kato, Commun. Pure Appl. Math., 10 (1951), 151. [2] S. Agmon, Lectures on Exponential Decay of Solutions of Second-Order Elliptical Equations: Bound on Eigenfunctions of N-Body Schrödinger Operators, Princeton University Press, 1982. [3] H.L. Kennedy, Y. Zhao, Int. J. Quantum Chem., 71 (1999), 1. [4] T. Özdoğan, M. Orbay, Int. J. Quantum Chem., 87 (2002), 15. [5] T. Özdoğan, Int. J. Quantum Chem., 92 (2003), 419. [6] J. Yasui, A. Saika, J. Chem. Phys., 76 (1982), 468. [7] T. Özdoğan, M. Orbay, Czechoslovak. J. Phys., 52:12 (2002), 1297-1302. [8] J. A. Pople, D. L. Beveridge, Approximate Molecular Orbital Theory, Mc-Graw Hill, 1970. [9] I. I. Guseinov, J. Phys. B, 3 (1970), 1399. [10] I. I. Guseinov, Int. J. Quantum Chem., 2003; DOI 10.1002/qua.10336. [11] M. P. Barnett, Theor. Chem. Acc., 107 (2002), 241. [12] E. Öztekin, M. Yavuz, S. Atalay, J. Mol. Struct. THEOCHEM, 536 (2001), 59. [13] I. I. Guseinov, Int. J. Quantum Chem., 67 (1998), 199.

т. Оздоган

Поступило

Университет Ондокуз Майис

03.08.2005

E-mail: telhatoz@omu.edu.tr

М. Орбей

Университет Ондокуз Майис

E-mail: morbay@omu.edu.tr 